\title{
Unbalanced GLA mRNAs ratio quantified by real-time PCR in Fabry patients' fibroblasts results in Fabry disease
}

\author{
Camilla Filoni ${ }^{1,8}$, Anna Caciotti ${ }^{1,8}$, Laura Carraresi ${ }^{1}$, Maria Alice Donati ${ }^{1}$, Renzo Mignani ${ }^{2}$, \\ Rossella Parini ${ }^{3}$, Mirella Filocamo ${ }^{4}$, Fausto Soliani ${ }^{5}$, Lisa Simi ${ }^{6}$, Renzo Guerrini ${ }^{1}$, \\ Enrico Zammarchi ${ }^{*, 7}$ and Amelia Morrone ${ }^{1}$
}

\footnotetext{
${ }^{1}$ Metabolic and Muscular Unit, Clinic of Pediatric Neurology, AOU Meyer, University of Florence, Florence, Italy; ${ }^{2}$ Nephrology Unit, Ospedale Infermi, Rimini, Italy; ${ }^{3}$ Metabolic Unit, San Gerardo Hospital, Monza, Italy; ${ }^{4}$ Diagnosi Pre-Postnatale Malattie Metaboliche, IRCCS Gaslini, Genoa, Italy; ${ }^{5}$ Nephrology Unit, Arciospedale S. Maria Ospedale Nuova, Reggio Emilia, Italy; ${ }^{6}$ Department of Clinical Physiopathology, University of Florence, Florence, Italy;

${ }^{7}$ Department of Pediatrics, University of Florence, Florence, Italy
}

Total or partial deficiency of the human lysosomal hydrolase $\alpha$-galactosidase $A$ is responsible for Fabry disease, the $X$-linked inborn error of glycosphingolipid metabolism. Together with the predominant $\alpha$-galactosidase A gene mRNA product encoding the lysosomal enzyme, a weakly regulated alternatively spliced $\alpha$-galactosidase A transcript is expressed in normal tissues, but its overexpression, due to the intronic g.9331G $>$ A mutation, leads to the cardiac variant. We report the molecular characterization of five Fabry patients including two siblings. Sequencing analysis of the $\alpha$-galactosidase $A$ gene coding region and intron/exon boundaries identified the new c.124A $>G$ (p.M42V) genetic lesion as well as a known deletion in three patients, whereas in the two remaining patients, no mutations were identified. To evaluate possible $\alpha$-galactosidase A gene transcription alterations, both predominant and alternatively spliced mRNAs were quantified by absolute real-time PCR on total RNA preparations from the patients' fibroblasts. An impressive reduction in the predominant $\alpha$-galactosidase $A$ transcript was detected in the last patients (Pt 4 and Pt 5). However, the alternatively spliced mRNA was dramatically overexpressed in one of them, carrying a new intronic lesion (g.9273C $>$ T). These findings strongly suggest a correlation between this new intronic mutation and the unbalanced $\alpha$-galactosidase A mRNAs ratio, which could therefore be responsible for the reduced enzyme activity causing Fabry disease. The real-time assay developed here to investigate the two $\alpha$-galactosidase A mRNAs might play a crucial role in revealing possible genetic lesions and in confirming the pathogenetic mechanisms underlying Fabry disease. European Journal of Human Genetics (2008) 16, 1311-1317; doi:10.1038/ejhg.2008.109; published online 18 June 2008

Keywords: Fabry disease; $\alpha$-galactosidase; cryptic exon

\footnotetext{
*Correspondence: Professor E Zammarchi, Department of Pediatrics, University of Florence, Via Luca Giordano 13, Florence 50132, Italy. Tel: + 39055 5662482; Fax: + 39055 570380;

E-mail: neuromet@meyer.it

${ }^{8}$ These authors contributed equally to the work

Received 10 January 2008; revised 29 April 2008; accepted 6 May 2008; published online 18 June 2008
}

Introduction

Anderson-Fabry disease (OMIM no. 301500) is the only known X-linked sphingolipid error of metabolism characterized by total or partial deficiency of the lysosomal hydrolase $\alpha$-galactosidase $(\alpha$-GalA, EC 3.2.1.22). Glycosphingolipids accumulate in the plasma and lysosomes of 
many tissues, culminating in renal, cardiac and cerebrovascular manifestations. ${ }^{1,2} \alpha$-GalA is encoded by the seven exon GLA gene (OMIM no. 300644), located at Xq22, and more than 450 genetic lesions have been identified as disease-causing mutations. ${ }^{1,3}$

The classic form of the disease represents the most severe phenotype, but less severe cardiac and renal variants have been identified. ${ }^{4-6}$ Affected male patients with milder variants usually show residual enzyme activity and have a normal life span with cardiac or renal symptoms only. ${ }^{1,7}$

Heterozygous female patients may develop moderateto-severe features as a result of random and uneven X-chromosomal inactivation. ${ }^{1}$ Despite the multisystemic involvement of the classic form of the disease, which results in a life span of about 40-50 years, the introduction of enzyme replacement therapy (ERT) ${ }^{8,9}$ has been demonstrated to prevent, arrest and potentially reverse disease progression. ${ }^{10}$

Diagnosis is often supported by a positive family history and is usually confirmed in male patients by $\alpha$-GalA assays on leukocytes or fibroblasts, and by GLA gene mutation analysis. $^{1}$

The cardiac variant of Fabry disease can be caused by missense mutations, small in frame deletions and by the mid-intronic genetic lesion g.9331G $>$ A (c.639 + 919G $>$ A) of the GLA gene. ${ }^{1,7}$ This latter mutation increases the recognition of a normally rare alternatively spliced GLA transcript that corresponds to the wild-type (WT) transcript with the insertion of $57 \mathrm{nt}$ from a cryptic exon in intron $4 .^{7}$ An increased recognition of the alternatively spliced transcript, due to the intronic variant g.9331G $>$ A mapped into the $3^{\prime}$ end of the $57 \mathrm{bp}$ cryptic exon, leads to an unbalanced WT/mutant transcript ratio (from about $1-10 \%$ in normal tissues to more than $70 \%$ detected in the mutant cells) resulting in lower $\alpha$-GALA activity. ${ }^{7}$

In summary, Fabry disease presents with a remarkable molecular heterogeneity due to the large number of mutations detected in the GLA gene, the spread of mutations throughout the entire gene and genomic variants involving alternative mRNA splicing. This study focuses on the quantification, by real-time PCR, of the alternatively spliced GLA mRNAs, helping to further widen the wide spectrum of genetic defects detectable in Fabry patients. The method developed here also represents a contribution to still limited data on the quantitative evaluations of mRNA transcripts in lysosomal storage diseases. $^{11-17}$

We also report the results of biochemical and sequencing analyses of five male Fabry patients.

\section{Materials and methods Specimens}

GLA mRNA analysis was performed in cultured fibroblasts derived from five Fabry male patients and 15 controls. Table 1 summarized patient (Pt) characteristics. Apart from the two brothers, Pt1 and Pt2, the remaining patients were unrelated. Pt1, Pt2 and Pt3 presented with classic phenotypes. Pt4 was a 64-year-old man at the time of our survey. At 13 years, he suffered from acroparesthesia, fever and pain crises. At 37 years, proteinuria was present, and after a renal biopsy, a diagnosis of Fabry disease was made. At 39 years, the patient underwent kidney transplantation, and at 52 years hypertrophic cardiomyopathy and cornea verticillata were present. The sister of Pt4 is a 61-year-old symptomatic female presenting with acroparesthesia, abdominal pain and osteoporosis. Pt5 is a 14-year-old man. His presenting symptoms, at the age of 9, were acroparesthesia, fever pain crises and cornea verticillata.

\section{Analysis of genomic DNA}

The entire coding region and exon boundaries of the GLA gene of the five Fabry patients were amplified by PCR. The oligonucleotides and the amplifying conditions have been described previously. ${ }^{7,18}$

The PCR products were checked on 2\% agarose gels, excised and purified using Nucleospin Extract kit (Macherey-Nagel, Düren, Germany). Sequencing reactions were performed using the ABI PRISM 310 Genetic Analyzer (Applied Biosystems, Foster City, USA), as recommended by the manufacturer.

Table 1 Biochemical and sequencing analyses of the GLA gene from fibroblasts of the six Fabry patients

\begin{tabular}{llllr}
\hline Patient & Sex & $\begin{array}{l}\text { GLA nucleotide } \\
\text { change }\end{array}$ & Effect on $\alpha$-GALA coding region & $\begin{array}{c}\text { Enzyme activity in fibroblasts } \\
(\mathrm{nmol} / \mathrm{mg} / \mathrm{hr}) \pm \text { SD }\end{array}$ \\
\hline 1 & Male & Ex3_4del & Deletion of exons 3 and 4 & $0.26 \pm 0.21$ \\
2 & Male (brother of & Ex3_4del & Deletion of exons 3 and 4 & $0.19 \pm 0.12$ \\
3 & Patient 1) & c.124A $>$ G & p.M42V & $0.09 \pm 0.13$ \\
4 & Male & g.9273C $>$ T & Enhancement of cryptic exon & $0.5 \pm 0.13$ \\
5 & Male & - & insertion & $0.34 \pm 0.3$ \\
\hline
\end{tabular}

Values are the average of three independent experiments performed in duplicate. Values outside the normal ranges are italicized. Normal value of $\alpha$-GALA enzyme activity: $25-75 \mathrm{nmol} / \mathrm{mg} / \mathrm{hr}$. 
Genomic DNA from the sister of Pt4 was isolated and tested to confirm the molecular data. All patients completed a consent form approved by the local Ethics Committee before genetic analyses were carried out.

\section{Restriction fragment length polymorphism (RFLP) analysis}

Mutation c.124A $>$ G (M42V) Exon 1 of the GLA gene was PCR-amplified in Pt3 and in 100 control alleles, as described previously. ${ }^{18}$ The new c. $124 \mathrm{~A}>\mathrm{G}$ (M42V) sequence variation was investigated by restriction analysis with the NcoI enzyme (Fermentas, Canada) whose canonical restriction site was lost in the mutated specimen.

Mutation g.9273C $>\boldsymbol{T}$ The specific fragment of intron 4 was PCR-amplified in Pt4, in his sister and in 100 control alleles, as described previously. ${ }^{7}$ The new g.9273C $>\mathrm{T}$ nucleotide change was investigated by the BseMII restriction enzyme that is present as a natural restriction site.

PCR products were run on $2 \%$ agarose gel. A $10 \mu \mathrm{l}$ aliquot of PCR products was incubated overnight at the temperature recommended by the manufacturer, with a reaction mixture containing $1.5 \mu \mathrm{l}$ of $10 \mathrm{X}$ reaction buffer and $5 \mathrm{U}$ of the specific restriction enzyme. The total volume was brought up to $20 \mu \mathrm{l}$. The NcoI-digested and BseMII-digested PCR products were analyzed on $2 \%$ agarose gel.

\section{RNA isolation and analyses}

All reagents for total RNA extraction from patients' fibroblasts were purchased from Eppendorf AG (Hamburg, Germany). RNA integrity and concentrations were checked by $0.8 \%$ agarose gel electrophoresis and Nanodrop ${ }^{\circledR}$ ND1000 Spectrophotometer. Controls and patient total RNAs (400 ng) were reverse transcribed in $80 \mu \mathrm{l}$ of final volume in a reaction mixture containing $10 \mu \mathrm{l}$ TaqMan RT buffer $1 \mathrm{X}$, $5.5 \mathrm{mM} \mathrm{MgCl}_{2}, 500 \mu \mathrm{M}$ each dNTP, $2.5 \mu \mathrm{M}$ random hexamers, $0.4 \mathrm{U} / \mu \mathrm{l}$ RNase inhibitor and $1.25 \mathrm{U} / \mu \mathrm{l}$ multiScribe reverse transcriptase provided by Applied Biosystems. The profile of the one-step reverse transcriptase was as follows: $10 \mathrm{~min}$ at $25^{\circ} \mathrm{C}, 30 \mathrm{~min}$ at $48^{\circ} \mathrm{C}$ and $2 \mathrm{~min}$ at $95^{\circ} \mathrm{C}$. Reversetranscriptase PCR (RT-PCR) was performed with the amplification primers described previously. ${ }^{18}$ Cycle conditions were the same as those used for the analysis of genomic DNA.

\section{Mutation nomenclature}

All mutations are described according to the mutation nomenclature (http://www.hgvs.org/mutnomen/recs.html) considering nucleotide +1 as the $\mathrm{A}$ of the first ATG translation initiation codon. ${ }^{19,20}$ Nucleotide numbers are derived from cDNA GLA sequence (EMBL/Gen Bank/DDBJ; accession number NM_000169) and from g.DNA GLA sequence (EMBL/Gen Bank/DDBJ; accession number X14448).

\section{Quantification of $\alpha$-GalA mRNA transcripts}

The measurement of both GLA gene mRNAs was performed using a quantitative real-time RT PCR method based on TaqMan $^{\mathrm{TM}}$ technology. Probes and primers were selected by the computer program 'Primer Express' (Applied Biosystems). For the detection of WT GLA mRNA, the following probe and primers were chosen on WT GLA cDNA (1290 bp):

probe 631/666 nt: 5' TTTCAAAAGCCCAATTATACAGA AATCCGACAGTAC 3' labeled with VIC, located on the junction region of exons 4 and 5 , forward primer 579/ 602 nt: 5' GACTGGCAGAAGCATTGTGTACTC 3', which hybridizes on exon 4, reverse primer 688/668 nt: $5^{\prime}$ CAAAATTTCGCCAGTGATTGC 3', located on exon 5.

For the detection of the alternative GLA gene mRNA, the following probe and primers were designed on the cDNA carrying the intronic insertion of 57 nts between exons 4 and 5 (1347 bp):

probe 670/701 nt: 5' CTTGTTACCATGTCTCCCCACT AAAGTCCCAA $3^{\prime}$ labeled with FAM, which hybridizes on the junction region between intronic insertion and exon 5 , forward primer 642/668 nt: 5' CTCCACACTATTTGGAAGT ATTTGTTG $3^{\prime}$, located on intronic insertion, reverse primer 738/718 nt: 5' TCGCCAGTGATTGCAGTACTG 3', located on exon 5.

PCR analysis was performed using $25 \mathrm{ng}$ of cDNA in a reaction mixture containing $300 \mathrm{nM}$ of forward and reverse primers and $200 \mathrm{~nm}$ of the fluorescent probes (for each gene in different wells), and $12.5 \mu$ l Universal master Mix. Plates were treated for $2 \mathrm{~min}$ at $50^{\circ} \mathrm{C}$ for $10 \mathrm{~min}$ at $95^{\circ} \mathrm{C}$ and then submitted to 40 cycles of amplification at $95^{\circ} \mathrm{C}$ for $15 \mathrm{~s}$ and $60^{\circ} \mathrm{C}$ for $1 \mathrm{~min}$ in the ABI Prism 7000 Sequence Detector PE (Applied Biosystems). Plasmid vector carrying GLA gene major transcript was generated by site direct mutagenesis and fragment replacement starting from a pCD-X vector kindly provided by Dr d'Azzo. For the absolute quantification of the alternatively spliced GLA mRNA, the following oligonucleotide was designed on the cDNA carrying the intronic insertion of $57 \mathrm{nt}(1347 \mathrm{bp})$ and generated by Roche, Mannheim, Germany: oligonucleotide GLA mut

5'CTCCACACTATTTGGAAGTATTTGTTGACTTGTTACCA TGTCTCCCCACTAAAGTCCCAATTATACAGAAATCCGACA GTACTGCAATCACTGGCGA 3' (642/738 nt)

Plasmid and oligonucleotides were serially diluted 10 fold from a starting quantity of $10^{8}$ ag for WT gene and $10^{7}$ ag for mutated gene expression analyses and used as standard curves. The absolute values of GLA gene mRNA products were expressed as copy/ $\mu$ g total RNA (mean $\pm \mathrm{SD}$ ).

\section{Statistical analysis}

Statistical analysis was carried out using the SPSS software package (SPSS Inc., Chicago, IL). Statistical differences between groups were assessed by $t$-test. Differences with $P<0.05$ were considered statistically significant. 


\section{Results}

\section{Molecular studies}

The fibroblasts from patients with enzymatic diagnosis of Fabry disease underwent molecular characterization (Table 1). The GLA gene exons and exon/intron boundaries were amplified and directly sequenced on both strands.

The new c. $124 \mathrm{~A}>\mathrm{G}$ nucleotide change that leads to a p.M42V amino-acid substitution was identified at the hemizygous level in male Pt3 (Table 1). PCR analysis of the genomic region encompassing exons 3 and 4 failed to reveal any genomic amplification products from the two siblings (Pt1 and Pt2). Instead, RT-PCR analysis on the same two patients showed an abnormally smaller product (609 bp) than the expected $880 \mathrm{bp}$, as shown in Figure 1. Sequencing analysis confirmed a $270 \mathrm{bp}$ deletion corresponding to the whole coding sequence of the exons 3 and 4. Such deletion has previously been described at a genomic level. ${ }^{21}$

Although no mutations were identified at the genomic level in the GLA gene coding region or intron/exon boundaries of Pt4 and Pt5 (Table 1), RT-PCR analysis showed in Pt4 an abundant presence of a longer fragment (939 bp) than the expected one $(882 \mathrm{bp})$. The latter was instead present in a very reduced amount in Pt5. No additional aberrant transcripts were detected (Figure 2). Sequencing analysis showed that the large transcript that was detected in Pt4 corresponds to the physiological, alternatively spliced GLA mRNA including the 57 intronic nucleotides. $^{7}$

Specific genomic sequencing analysis of the intron 4 region, encompassing the 57 nucleotides (g.9278_9334) cryptic exon region, was also performed in both specimens (Pt4 and Pt5). A new intronic nucleotide variation g.9273C $>$ T (c.861-5 C > T), mapping $5 \mathrm{nt}$ upstream of the 3 'ending of the cryptic exon, was identified in Pt4 (Table 1) (Figure 3).

No other mutations, including the g.9331G $>$ A genetic lesion, previously associated to the increased recognition of a normally weak splice site, ${ }^{7}$ were found.

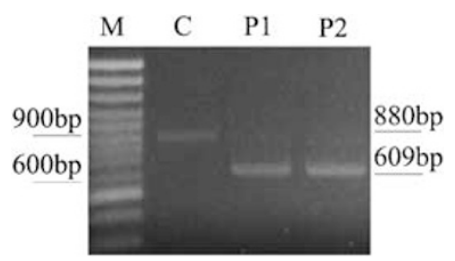

Figure 1 Identification of the Ex3_4 deletion. RT-PCR amplification was performed starting from $400 \mathrm{ng}$ of total RNA, prepared from Pt1 and Pt2 fibroblasts and from normal fibroblasts. The primers used encompass the GLA gene mRNA region between exons 1 and 7 . The oligonucleotides used were reported previously. ${ }^{18}$ The normal pattern shows the $880 \mathrm{bp}$ fragment, whereas the mutated hemizygous pattern shows the $609 \mathrm{bp}$ corresponding to such a region missing exons 3 and 4. C, normal control; P1, patient 1P2; patient 2; M, molecular weight marker.

The possible polymorphic nature of the new point genetic changes c.124A $>$ G (p.M42V) and g.9273C $>$ T was excluded by RFLP analysis ascertaining that none of the 50 healthy female controls carried these alterations. In addition, the new g.9273C $>\mathrm{T}$ intronic nucleotide variation was also identified in the affected sister of Pt4.

\section{Real-time detection of the $G L A$ gene mRNA variants} Quantitative analyses of GLA gene mRNA products were performed by absolute real-time PCR. To determine the quantity of mRNA in patients' specimens, pCD-GLA vector and oligonucleotide-GLAmut were used to generate the standard curves. Tenfold dilution series of both plasmid and oligonucleotide were tested to obtain the dynamic ranges. The results reported in Figures 4 and 5 indicated that these assays effectively distinguished 10-fold differences in concentration from about 20 to $2 \times 10^{6}$ templates' $^{\prime}$ molecules per reaction mixture (in WT GLA assay) and from $1.9 \times 10^{3}$ to $1.9 \times 10^{8}$ templates' molecules per reaction mixture (in mutant GLA assay).

We analyzed the total RNA isolated from the fibroblasts of five male patients (including the two siblings Pt1 and Pt2) and 15 normal controls.

The results of the quantification of GLA gene mRNA variants are summarized in Table 2 . With respect to the SD,

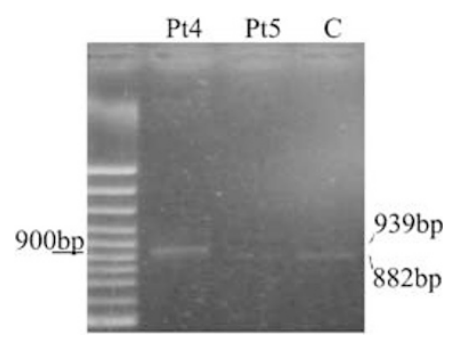

Figure 2 Identification of the alternatively spliced mRNA in fibroblasts from Pt4. RT-PCR analysis was performed starting from $400 \mathrm{ng}$ of total RNA, prepared from Pt4 and Pt5 fibroblasts and from normal fibroblasts. The primers used encompass the GLA gene mRNA region between exons 1 and 7 . The oligonucleotides used were reported previously. ${ }^{18}$ The normal pattern shows the 882 bp fragment, whereas the mutated hemizygous pattern shows the $939 \mathrm{bp}$ corresponding to such a region with the insertion of $57 \mathrm{nt}$ from intron 4. C, normal control; Pt4, patient 4; Pt5, patient 5.

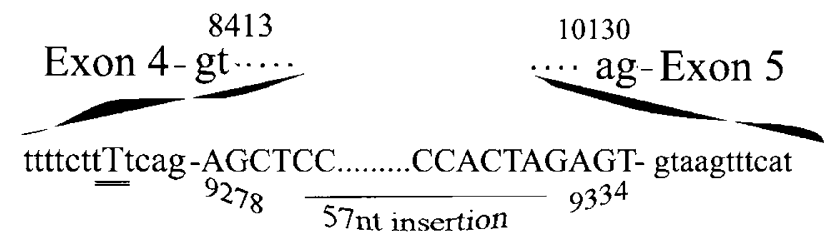

Figure 3 Graphic representation of the g.9273C $>$ T intronic lesion into the partial sequence of $\alpha$-Gal A intron 4 from Pt4. C $>$ t transition at $9273 \mathrm{nt}$ is indicated by double underlining. Pt4, patient 4 . 


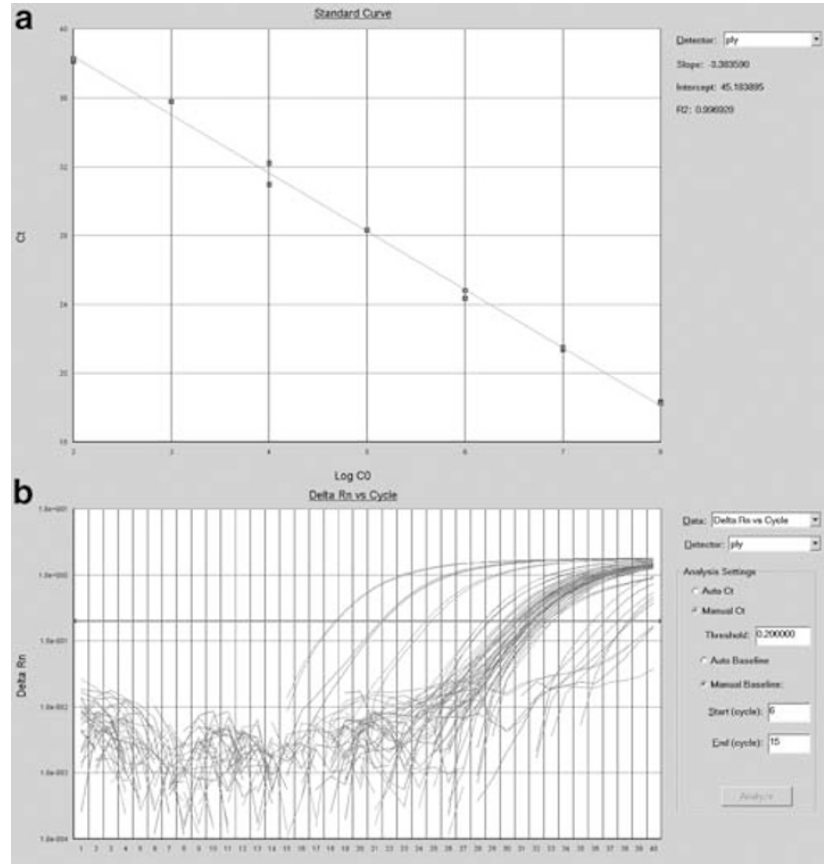

Figure 4 Real-time analysis of GLA gene wild-type mRNA. (a) Standard curve was generated by seven 10 -fold serial dilutions of pCD-GLAWT from a starting quantity of $2 \times 10^{6}$ standard copies. (b) Amplification plot.

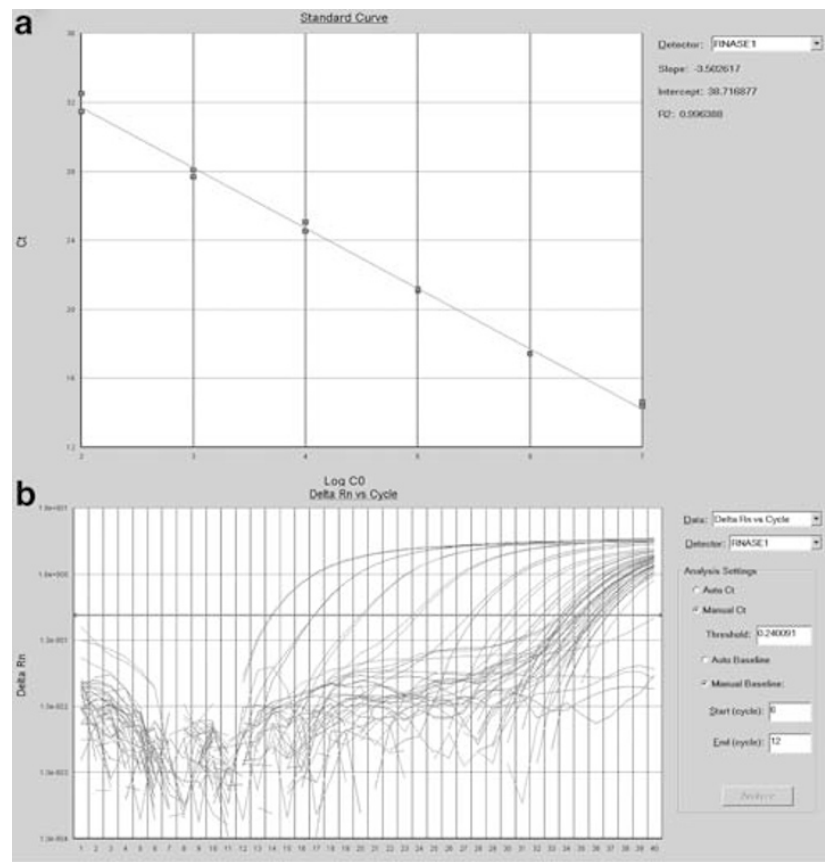

Figure 5 Real-time analysis of GLA gene mutated mRNA. (a) Standard curve was generated by six 10 -fold serial dilutions of oligonucleotide GLAmut from a starting quantity of $1.9 \times 10^{6}$ standard copies. (b) Amplification plot.
Table 2 Number of GLA mRNA copies detected in Fabry patients and control samples

\begin{tabular}{lcc}
\hline Patient & $\begin{array}{c}\text { Number of GLA WT } \\
\text { copies } \pm S D\end{array}$ & $\begin{array}{c}\text { Number of GLAmut } \\
\text { copies } \pm S D\end{array}$ \\
\hline 1 and 2 & $\mathrm{ND}$ & \multicolumn{1}{c}{$\mathrm{ND}$} \\
4 & $3.61 \mathrm{E}+04 \pm 9.4 \mathrm{E}+03$ & $2.18 \mathrm{E}+05 \pm 1.1 \mathrm{E}+05$ \\
5 & $3.69 \mathrm{E}+04 \pm 1.00 \mathrm{E}+04$ & $5.28 \mathrm{E}+03 \pm 2.3 \mathrm{E}+03$ \\
$\begin{array}{l}\text { Normal mean } \\
\text { value }\end{array}$ & $1.40 \mathrm{E}+05 \pm 7.26 \mathrm{E}+04$ & $1.00 \mathrm{E}+04 \pm 9.66 \mathrm{E}+03$ \\
\hline
\end{tabular}

Patients with values outside the mean are reported in detail.

Values are the average of three independent experiments performed in duplicate. Values outside the normal ranges are italicized. ND, not detectable.
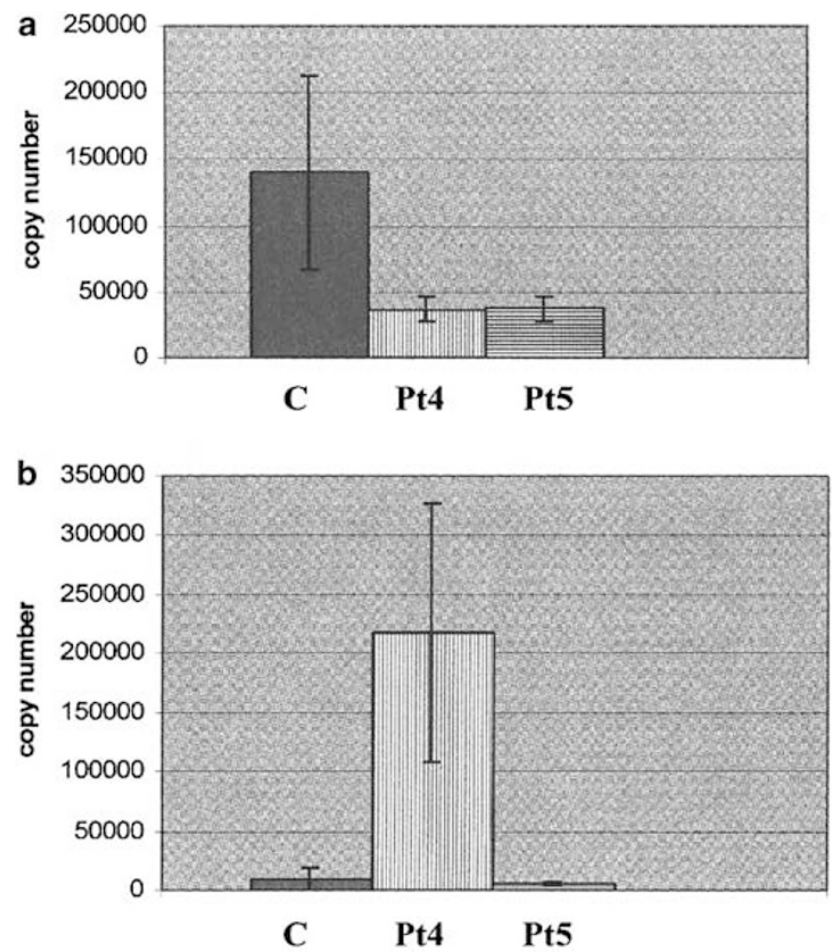

Figure 6 Graphic representation of Real-time RT-PCR of the controls and Pt4 and Pt5 with values outside the mean. (a) Number of GLA mRNA wild-type copies detected in controls (C) and in Fabry Pt4 and Pt5. (b) Number of alternatively spliced GLA mRNA copies detected in controls (C) and in Fabry Pt4 and Pt5. Pt4, patient 4; Pt5, patient 5.

the mean value of WT GLA control mRNA was not significantly different from that of Pt3. Neither were there significant differences between the mutant GLA mRNA levels of this patient and controls. In contrast, the two patients carrying the deletion (Pt1 and Pt2) showed a complete absence of both mRNAs because of impaired annealing of the Taq-Man probes or primers mapping between exons 4 and 5 . Interestingly, samples from Pt4 and Pt5 both showed reduced amounts of GLA major transcript 
as compared to the control values (Figure 6a) (Table 2). In addition, the GLA gene alternatively spliced product was greatly enhanced in the specimen from Pt4 carrying the g.9273C $>\mathrm{T}$ intronic mutation (Table 2) (Figure 6b).

The splice site score calculation resulting from the http:// www.fruitfly.org/ showed that the acceptor predict score of 0.98 between the WT and mutant g.9273C > T cryptic exon remains unchanged.

The online tool ACESCAN2 Web Server (http://genes. mit.edu/acescan2/index.html) used to identify cis-element candidates in alternative and constitutive splicing of mammalian exons predicted the creation of a new exonsplicing enhancer (ESE) by the new g.9273C $>\mathrm{T}$ intronic mutation.

\section{Discussion}

Mutations in the GLA gene are very heterogeneous and most of them are private; however, mutation detection may be important for confirming diagnosis and genotypephenotype correlation, and it represents the only certain method to identify heterozygous female patients and to introduce early treatment with ERT in Fabry patients. Knowing the genetic lesions is also crucial for the application of the emerging therapeutic strategy using small molecules termed active-site-specific chaperones (ASSC) that are 'pharmacological chaperones'. These small molecules can not only be delivered orally to reach all tissue types, but also they have the ability to increase the residual enzyme activity. ${ }^{22,23}$

We contributed to the Fabry mutation database in identifying a new point mutation c. $124 \mathrm{~A}>\mathrm{G}$ (p.M42V) in the fibroblasts of one Fabry patient analyzed.

Molecular characterization using genomic DNA standard protocols as sequencing analysis failed to identify any mutation in Pt4 and Pt5, who exhibited classic phenotypes and very low GLA enzyme activity. In Pt4, GLA mRNA RTPCR showed an abundant transcript with 57 nt insertion, which corresponds to the normally rare alternatively spliced GLA transcript. ${ }^{7}$ This finding has previously been ascribed to the presence of the g.9331G $>$ A genetic lesion that caused an unbalanced ratio of the normal/mutated mRNAs. ${ }^{7}$ Such mutation enriches the A/C predominance for a $57 \mathrm{nt}$ sequence of intron 4 , acting as an exonic splicing enhancer and leading to the cardiac variant of Fabry disease with residual enzyme activity. ${ }^{7}$ With respect to the $57 \mathrm{nt}$ cryptic exon, Pt4 presented the g.9273C $>\mathrm{T}$ intronic nucleotide substitution $5 \mathrm{nt}$ upstream to the alternative $3^{\prime}$ splicing junction. This genetic lesion is absent in the normal population and in all analyzed patients, but it is present in his affected sister.

A new ESE with the unchanged acceptor score was predicted to be caused by the new g.9273C $>$ T intronic substitution. This is consistent with our data and might confirm the key role of this nucleotide substitution in the improvement of the alternative GLA splicing.

To investigate the molecular defect identified in Pt4, we focused our work on the development of GLA gene quantitative expression profiling. Such analysis produced fast and accurate results on the quantity of both the GLA mRNAs: the WT transcript, resulting in the lysosomal enzyme, and the normally weak expressed alternative product. In the normal controls, in agreement with existing literature, ${ }^{7}$ the expression level of the alternatively spliced mRNA was about $7 \%$ of the normally spliced product. Pt4 and Pt5, with no mutations in the GLA coding region, presented with abnormal transcript levels. Both patients presented with reduced amounts of the WT transcript, suggesting an alteration in the synthesis or processing of the GLA mRNA, or both. More interestingly, Pt4 showed an enormously increased level of the alternatively spliced mRNA. Moreover, the copy number of this transcript was greater than that of normal GLA mRNA, suggesting that in this patient's fibroblasts, the normally rare splicing event occurs as the more frequent variant. In contrast with patients described by Ishii et $\mathrm{al}^{7}$ as carrying g.9331G $>A$ and affected by the Fabry disease cardiac variant with reduced $\alpha$-GALA activity $(10 \%$ of normal controls), Pt4 presents with the classic phenotype and a very low residual enzyme activity. Thus, the unbalanced ratio of the GLA mRNAs and the relative expression levels of both transcripts in the tissues can be considered responsible for very different clinical presentations and low $\alpha$-GALA enzyme activities. The genetic causes of this alternative splicing event are variable and should be carefully investigated at clinical, biochemical and molecular levels.

Our findings show that an atypical molecular pattern can be also observed in patients with the classical form of the disease. However, additional molecular data are needed to explain the findings of Pt5 having no mutations in the GLA coding region, the reduced amount of WT GLA mRNA and normal expression of the mutated transcript.

In conclusion, we provide additional evidences on the remarkable genetic variability of Fabry disease, addressing the development of a key protocol to improve the rapid and accurate mutation detection in such patients. Standard molecular procedures in mutation detection may fail to reveal any genetic lesions in the GLA coding region and the intron/exon boundaries of patients' specimens. Mutation detection is important for diagnosis confirmation and genotype-phenotype correlation. The real-time PCR assays devised here can absolutely quantify the molecular defect (transcriptional alterations) of both GLA gene and mRNAs in Fabry patients, quickening genetic diagnosis within family units. 


\section{Acknowledgements}

The cell lines of the two brothers and two additional patients, one male and one female, with the classic phenotype were obtained from the 'Cell Line and DNA Bank from Patients Affected by Genetic Diseases' collection (http://www.gaslini.org/labdppm.htm). This paper was partially financed by grants: Genzyme grant n.104 19/2007, Fondi Ateneo (MURST ex 60\%). We gratefully thank Professor Claudio Orlando from the Department of Clinical Physiopathology University of Florence, Florence, Italy, for the technical support for real-time PCR setting-up and a critical discussion of the manuscript.

\section{References}

1 Desnick RJ, Ioannou YA, Eng CM: Fabry disease: $\alpha$-galactosidase A deficiency; in Scriver C, Beaudet A, Sly W, Valle D (eds): The Metabolic and Molecular Bases of Inherited Disease, 7th edn. New York: McGraw-Hill, 2001, pp 3733-3774.

2 Desnick RJ, Brady R, Barranger J et al: Fabry disease, an underrecognized multysistemic disorder: export recommendations for diagnosis, management, and enzyme replacement therapy. Ann Intern Med 2003; 138: 338-346.

3 Shabbeer J, Yasuda M, Benson SD, Desnick RJ: Fabry disease: Identification of 50 novel $\alpha$-galactosidase A mutations causing the classic phenotype and three-dimensional structural analysis of 29 missense mutations. Hum Genomics 2006; 2: 297-309.

4 Elleder M, Bradova V, Smid F et al: Cardiocyte storage and hypertrophy as a sole manifestation of Fabry's disease. Report on a case simulating hypertrophic non-obstructive cardiomyopathy. Virchows Arch A Pathol Anat Histopathol 1990; 417: 449-455.

5 Frustaci A, Chimenti C, Ricci R et al: Improvement in cardiac function in the cardiac variant of Fabry's disease with galactoseinfusion therapy. N Engl J Med 2001; 345: 25-32.

6 Nakao S, Kodama C, Takenaka T et al: Fabry disease: detection of undiagnosed hemodialysis patients and identification of a 'renal variant' phenotype. Kidney Int 2003; 64: 801-807.

7 Ishii S, Nakao S, Minamikawa-Tachino R, Desnick RJ, Fan JQ: Alternative splicing in the alpha-galactosidase A gene: increased exon inclusion results in the Fabry cardiac phenotype. Am J Hum Genet 2002; 70: 994-1002.

8 Eng CM, Guffon N, Wilcox WR et al: Safety and efficacy of recombinant human alpha-galactosidase A replacement therapy in Fabry's disease. N Engl J Med 2001; 345: 9-16.

9 Schiffmann R, Kopp JB, Austin III HA et al: Enzyme replacement therapy in Fabry disease: a randomized controlled trial. JAMA 2001; 285: 2743-2749.

10 Desnick RJ, Brady RO: Fabry disease in chilhood. J Pediatr 2004; 144: S20-S26.
11 Potratz A, Huttler S, Bierfreund U, Proia RL, Suzuki K, Sandhoff K: Quantification of m-RNAs encoding proteins of the glycosphingolipid catabolism in mouse models of GM2 gangliosidoses and sphingolipid activator protein precursor (prosaposin) deficiency. Biochim Biophys Acta 2000; 502: 391-397.

12 Rice JE, Sanchez JA, Pierce KE, Wangh LJ: Real-time PCR with molecular beacons provides a highly accurate assay for detection of Tay-Sachs alleles in single cells. Prenat Diagn 2002; 22: $1130-1134$

13 Hermans MM, van Leenen D, Kroos MA et al: Twenty-two novel mutations in the lysosomal alpha-glucosidase gene (GAA) underscore the genotype-phenotype correlation in glycogen storage disease type II. Hum Mutat 2004; 23: 47-56.

14 Dhami R, Passini MA, Schuchman EH: Identification of novel biomarkers for Niemann-Pick disease using gene expression analysis of acid sphingomyelinase knockout mice. Mol Ther 2006; 13: $556-564$

15 Lualdi S, Di Rocco M, Corsolini F et al: Identification of nine new IDS alleles in mucopolysaccharidosis II. Quantitative evaluation by real-time RT-PCR of mRNAs sensitive to nonsense-mediated and nonstop decay mechanisms. Biochim Biophys Acta 2006; 1762: $478-484$

16 Lualdi S, Pittis MG, Regis S et al: Multiple cryptic splice sites can be activated by IDS point mutations generating misspliced transcripts. J Mol Med 2006; 84: 692-700.

17 Caciotti A, Donati MA, Procopio E et al: GM1 gangliosidosis: molecular analysis of 9 patients and development of an RT-PCR assay for GLB1 gene expression profiling. Hum Mut 2007; 28: 204.

18 Morrone A, Cavicchi C, Bardelli T et al: Fabry disease: molecular studies in Italian patients and $\mathrm{X}$ inactivation analysis in manifesting carriers. J Med Genet 2003; 40: e103.

19 den Dunnen JT, Antonarakis SE: Mutation nomenclature extensions and suggestions to describe complex mutations: a discussion. Hum Mutat 2000; 15: 7-12.

20 den Dunnen JT, Paalman MH: Standardizing mutation nomenclature: why bother? Hum Mutat 2003; 22: 181-182.

21 Kornreich R, Bishop DF, Desnick RJ: Alpha-galactosidase A gene rearrangements causing Fabry disease. Identification of short direct repeats at breakpoints in an Alu-rich gene. J Biol Chem 1990; 265: 9319-9326.

22 Shin SH, Murray GJ, Kluepfel-Stahl S et al: Screening for pharmacological chaperones in Fabry disease. Biochem Biophys Res Commun 2007; 359: 168-173.

23 Ishii S, Chang HH, Kawasaki K et al: Mutant alpha-galactosidase A enzymes identified in Fabry disease patients with residual enzyme activity: biochemical characterization and restoration of normal intracellular processing by 1-deoxygalactonojirimycin. Biochem J 2007; 406: 285-295. 